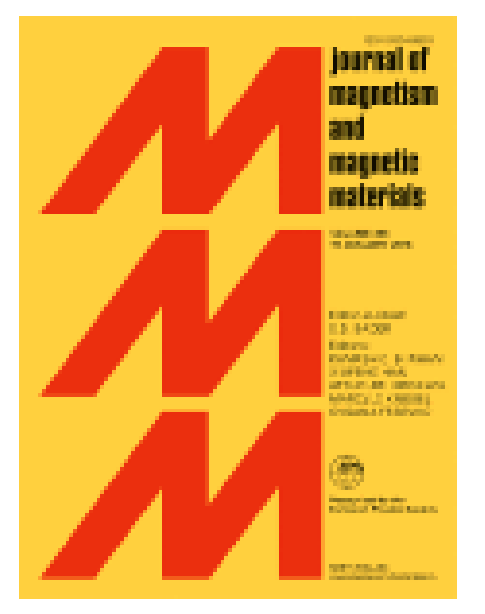

Article: "Measurement of damping in magnetic materials by optical heterodyne interferometry"

Authors: Chicharro J.M., Salazar F. y Bayón A.

Journal of Magnetism and Magnetic Materials, 268(3), pp. 348-356 (2004)

Keywords:

damping, elastic constants, resonance, interferometry, speckle

doi:10.1016/S0304-8853(03)00546-8 


\title{
MEASUREMENT OF DAMPING IN MAGNETIC MATERIALS BY OPTICAL HETERODYNE INTERFEROMETRY
}

\author{
J.M. CHICHARRO, A. BAYÓN, and F. SALAZAR \\ Dept. de Física Aplicada a los Recursos Naturales \\ E.T.S.I.Minas(Universidad Politécnica de Madrid) \\ c/Ríos Rosas, 21, 28003- Madrid (Spain) \\ e-mail: jchicharro@dfarn.upm.es
}

May 6, 2003

\begin{abstract}
A study of damping and its field-dependence in magnetic materials is presented. An optical heterodyne interferometer is used as detector of the longitudinal vibration of a slender rod located within a solenoid. Two different experiments are carried out in order to investigate damping in the demagnetized and saturated states. In one, the attenuation constant is determined by examining the free vibration of the sample. In the other, damping is indirectly measured from the sharpness of its resonance curve. Logarithmic decrement and its variation with the magnetic field is calculated. The detection and excitation systems used do not interact with the sample. Nickel rods and wires ranging in diameter from $2 \mathrm{~mm}$ to $10 \mathrm{~mm}$ are used as samples. Young's modulus is also determined.
\end{abstract}

Keywords: damping, elastic constants, resonance, interferometry, speckle PACS codes: 75.80.+q, 62.40.+i, 43.20.Ks, 43.35.Cg

\section{Introduction}

When materials are set in vibration some of the elastic energy is converted into heat. Losses can be evaluated by the ratio $\Delta W / W$, where $\Delta W$ is the energy dissipated in taking a specimen through a stress 
cycle and $W$ is the elastic energy stored in the specimen when strain is a maximum. This ratio is found to depend on amplitude, velocity, frequency, and type of vibration, as well as, other factors related to the past history of the sample and the temperature.

A measure of damping of elastic waves is the ratio between successive free oscillations. The natural logarithm of that ratio is called the logarithmic decrement $\delta$, which is defined as

$$
\delta=\frac{1}{n} \ln \left(\frac{A_{i}}{A_{i+n}}\right),
$$

where $A_{i}$ and $A_{i+n}$ are the amplitudes for the oscillation $i$ and $i+n$, respectively. Another measure of damping is given by the sharpness of the resonance curve under forced vibration. The full width of this curve at half-maximum amplitude $\Delta f_{a}$ is [1]

$$
\Delta f_{a}=\sqrt{3} f_{n} Q^{-1},
$$

where $f_{n}$ is the resonance frequency and $Q$ is the so-called quality factor. The above-mentioned damping parameters and $Q$ are related by

$$
\frac{1}{2 \pi} \frac{\Delta W}{W}=Q^{-1}=\frac{1}{Q} \simeq \frac{\delta}{\pi},
$$

the latter approximation can only be applied to cases of low internal friction $\left(Q^{-1}<<1\right)$; for $Q^{-1}=0.1$ the error is smaller than $5 \%$ [2].

As in any material, there are losses connected with the propagation of elastic waves through magnetic materials. However, for these materials damping is larger due to the magnetization process. Magnetic losses [1] are caused by macro and micro eddy currents and hysteresis. The latter results from irreversible displacement of domain walls and the former from changes of magnetization caused by the travelling wave. As is already known, damping is smaller for high magnetic fields due mainly to the fact that walls of magnetic domains are reorganized and magnetic directions remain stable.

There are several experimental methods [2] for measuring damping. Wave propagation methods are based on the measurement of the attenuation of a stress pulse travelling through the material. Forcedvibration experiments yield a resonance curve whose bandwidth is a measure of the internal friction. For free vibration, the method of obtaining damping involves the measurement of the free decay of vibration. The torsional pendulum is widely used for measuring internal friction. In this method, the sample, in the form of a wire, provides the restoring torque that governs the torsional oscillation of a suitable load. Examples of the application of these methods to magnetic materials are to be found in the publications [3], [4].

Speckle interferometry has been applied to elastically characterize non-magnetic isotropic samples [5], [6]. In more recent works [7], [8], this technique was used to measure the variation of the Young's 
modulus, $\Delta E$, and shear modulus, $\Delta G$, with the magnetic field $H$. The results obtained show that the compressibility modulus $\kappa$ remains constant with the magnetic field. In the present study, speckle interferometry is also used to detect the out-of-plane component of the vibration. The aim here is to measure damping in ferromagnetic materials in the form of rods and wires. In a first experiment, the sample is excited by an impact; the oscillogram of the decaying oscillations for the free longitudinal vibration is obtained and the logarithmic decrement determined. The method followed also permits us to know how damping affects Young's modulus. In another experiment, forced longitudinal vibration is induced using an electromagnetic driver that excites the sample without contact. As indicated above, the resonance curve yields the value of damping.

Knowledge of the behaviour of vibrating machinery is becoming increasingly important. Magnetic materials are widely used in engine manufacture. Systems such as transformers and, in general, electric engines work under the influence of magnetic fields. The fact that elastic properties and damping depend on magnetic fields results in a variation of resonance frequencies. Therefore, determination of elastic properties and damping of magnetic materials, along with their variation with the magnetic field, is of great interest. The method applied here is based on using a laser interferometer as a detector of the vibration of the sample. Thus, detection is point-like and without contact. The resolution of the system is high and its bandwidth broad. The system is heterodyne and, therefore, immune to low frequency vibration. The influence of the method followed to induce vibration in the sample on the magnetization process is negligible. A slender rod is used as the sample, which does not require complex preparation.

\section{Experimental set-up and free-vibration method}

The experimental set-up is shown in Fig.1. The studied samples are ferromagnetic wires and slender rods horizontally placed and supported at their centre on a small rubber block. The centre is a node for the lowest longitudinal mode. The sample constitutes the nucleus of a straight solenoid powered by a DC generator that also stabilizes the current intensity. The resulting magnetic field in the sample causes the sample to be magnetized almost longitudinally. The field is a function of the current carried by the solenoid and can be considered practically uniform. With this system, magnetic internal fields of up to 600 Oe can be obtained, which is sufficient to saturate the samples used in our experiments. Previous experiments [7] show that the rubber support prevents the sample from displacements due to the magnetic field and its effect on the longitudinal vibration is negligible. The ensemble solenoid plus sample are supported by a positioning system to correctly situate the sample opposite to the interferometer and to enable the selection of the point of detection.

Vibration in the sample is induced by a brief impact perpendicular to the base of the cylinder at its 
center. A pendulum with a quartz sphere whose diameter ranges from 1.80 to $4.40 \mathrm{~mm}$, depending on the frequency to be excited, is used to apply the impact. This type of excitation allows the sample to vibrate freely in its longitudinal modes, as the sample is isolated after the impact. Moreover, several longitudinal modes are simultaneously excited since the excitation spectrum has a broad bandwidth. The resulting free vibration is detected by registering the out-of-plane component of the displacement at the center of the opposite base. Damping in the material is determined by examining the variation of the amplitude of vibration. Spectral analysis of the detected displacements yields the natural frequencies of the sample.

A laser interferometer (OP-35-I/O from Ultra-Optec Inc.) is used to measure vibration of the sample at the detection point. The detection principle is based on speckle interferometry [9, 10]. Speckle phenomenon is observed when coherent light is scattered from a rough surface, resulting in an intensity distribution with bright and dark spots. Vibration at the detection point produces a variation in the optical path and, consequently, a phase change. With this interferometer, both displacement components, i.e. the out-of-plane and in-plane component, can be sequentially measured at the same point. This system has a bandwidth from $1 \mathrm{kHz}$ to $35 \mathrm{MHz}$ and a limit of detection of approximately $1 \mathrm{~nm}$. Due to the operation principle, the sample must have a diffusing surface, otherwise it should be scratched properly with sandpaper.

The optical unit includes a $5 \mathrm{~mW}$ He-Ne laser. The laser beam is divided in two rays by a Bragg cell; one having the same frequency as the original beam and the other with a frequency shifted by $40 \mathrm{MHz}$. In the out-of-plane configuration, which is used in our experiments, the unshifted frequency beam is focused on the surface of the sample at the detection point. The scattered light is collected in a direction symmetrical to that of the incident beam and led to the beam mixer where it interferes with the frequency-shifted beam used as a reference. Then, the resulting interference is focused on a photodiode.

A signal with a carrier frequency of $40 \mathrm{MHz}$, modulated in phase by the displacement of the detection point, is obtained at the output of the photodiode. This signal is demodulated in the electronic unit to yield a signal proportional to the instantaneous displacement. Finally, the signal is digitized using a Tektronic TDS-430A oscilloscope; with a sampling frequency twice the maximum frequency expected.

In studying propagation of a stress wave through an elastic solid, it is usually assumed that stress is proportional to strain. Due to damping of the stress wave, dissipative forces, which are usually assumed to be proportional to the velocity, should be included in the study. Like in any solid, elastic waves are damped when travelling through a magnetic material. There is a specific contribution to the damping associated with the magnetization process. Stress acting on a section will be given by the sum of three terms: an elastic term proportional to strain, a viscous term proportional to the variation in time of strain, and a magnetoelastic term related to the magnetic field applied [3]. Axial stress $\tau_{z z}$, the rotation 
axis of the rod is coincident with $o z$, can be expressed as

$$
\tau_{z z}=E_{H} \frac{\partial u_{z}}{\partial z}+\eta \frac{\partial}{\partial t}\left(\frac{\partial u_{z}}{\partial z}\right)-\frac{\lambda_{s} \chi E_{H}}{M_{s}} H_{z}
$$

where the symbols are as follows: $\eta$ damping constant, $E_{H}$ Young's modulus for the magnetic field $H_{z}, \lambda_{s}$ saturation magnetostriction, $\chi$ magnetic susceptibity, $M_{s}$ saturation magnetization, $u_{z}$ axial displacement, and $\frac{\partial u_{z}}{\partial z}$ axial strain. The general differential equation for longitudinal waves in slender rods is $\frac{\partial \tau_{z z}}{\partial z}=\rho \frac{\partial^{2} u_{z}}{\partial t^{2}}$, where $\rho$ is the density of the material. For this case, the differential equation that governs the propagation of damped longitudinal waves in magnetic rods will be obtained by substituting the value of $\tau_{z z}$ given by Eq.(4) into the above general equation, which results in the following motion equation

$$
\frac{\partial^{2} u_{z}}{\partial z^{2}}=\frac{\rho}{E_{H}} \frac{\partial^{2} u_{z}}{\partial t^{2}}-\frac{\eta}{E_{H}} \frac{\partial^{3} u_{z}}{\partial z^{2} \partial t}+\frac{\lambda_{s} \chi}{M_{s}} \frac{\partial H_{z}}{\partial z}
$$

In this case $H_{z}$ is approximately uniform, therefore, $\partial H_{z} / \partial z=0$. However, in each stage $H_{z}$ is changed. In order to find a solution to Eq.(5) for the free vibration, the method of separation of variables is applied. Substituting into the motion equation, Eq.(5), a solution of the form $u_{z}(z, t)=Z(z) \cdot T(t)$, we obtain [11]

$$
u_{z}(z, t)=(C \cos (k z)+D \operatorname{sen}(k z)) e^{-\frac{\gamma}{2} t}\left(A \cos \left(\omega_{l} t\right)+B \operatorname{sen}\left(\omega_{l} t\right)\right)
$$

where the attenuation constant $\gamma$ is $\omega_{0}^{2} \eta / E_{H}, \omega_{l}$ is equal to $\sqrt{\omega_{0}^{2}-\left(\frac{\gamma}{2}\right)^{2}}, \omega_{0}$ being the angular natural frequency for the undamped longitudinal vibration, $k$ is the wave number, and the $A, B, C, D$ constants depend on the initial and boundary conditions. Natural frequencies are calculated by applying the freeend conditions. For the damped longitudinal vibrations, natural frequencies $f_{l}$ are thus given by

$$
f_{n l}=\sqrt{\frac{n^{2}}{4 l^{2}}\left(\frac{E_{H}}{\rho}\right)-\frac{\gamma^{2}}{16 \pi^{2}}},
$$

where $n$ is a integer number associated with each normal mode.

Logarithmic decrement $\delta$ can be determined from $\gamma$ since the amplitudes $A_{i}$ and $A_{i+n}$, corresponding to the cycle number $i$ and $i+n$, respectively, are related by $A_{i+n}=A_{i} e^{-\frac{\gamma}{2} n T}, T$ being the period of vibration. Substituting this expression into Eq.(1), we obtain that $\delta$ can be calculated by

$$
\delta=\frac{\gamma}{2 f_{1 l}},
$$

where $f_{1 l}$ is the frequency for the first damped longitudinal mode. Eq.(8) provides the value of $\delta$ as a function of $\gamma$, associated with the dissipative term, and the first longitudinal frequency.

It is known from vibration theory, that for viscous damping, the envelope of the vibration is an exponential function. $\gamma$ is thus determined by fitting an exponential function to the peaks of the freedamped vibration. Fig. 2 shows the oscillograph records of the free vibrations for a Ni sample 180 $\mathrm{mm}$ in length and $10 \mathrm{~mm}$ in diameter in the demagnetized and saturated states. This figure illustrates 
that damping varies with magnetization, damping being larger in the demagnetized state, as expected. Both signals have been digitized using the previously mentioned Tektronic TDS-430A oscilloscope with a sampling frequency of $100 \mathrm{ksa} / \mathrm{s}$ and a number of samples equal to 15000. In Fig.2 are also plotted the exponential functions obtained in fitting such curves to the experimental data. The values of $\gamma$ are 102 $\mathrm{Hz}$ and $35 \mathrm{~Hz}$ for the demagnetized and technical saturated states, respectively.

Natural frequencies of the detected signals are calculated using the FFT. This option is included in the Tektronik oscilloscope used. As an example, Fig.3 shows the spectrum for the free-damped vibration of Fig. 2a. The first frequencies are $f_{1 l}=13580 \mathrm{~Hz}, f_{2 l}=27170 \mathrm{~Hz}$, and $f_{3 l}=40610 \mathrm{~Hz}$. The amplitude for the first mode is much larger than for the others. From the values of $f_{1 l}=13580 \mathrm{~Hz}$ and $\gamma=102$, Eq.(8) gives the logarithmic decrement $\delta$, for this case equal to $\delta=3.75 \cdot 10^{-3}$.

Eq.(7) provides the value of Young's modulus if $\rho, f_{1 l}, l$, and $\gamma$ are known. Using the first frequency, the expression for the calculation of $E$, when losses in the material are taken into account, is given by

$$
E=4 l^{2} \rho\left(f_{1 l}^{2}+\gamma^{2} / 16 \pi^{2}\right) .
$$

\section{$3 \quad$ Experimental results}

The samples studied were nickel rods and a nickel wire. The rods are $10 \mathrm{~mm}$ and $8 \mathrm{~mm}$ in diameter and 180 and $100 \mathrm{~mm}$ in length, respectively. The wire has a diameter of $2 \mathrm{~mm}$ and a length of 50 $\mathrm{mm}$. The metallographic analysis gives a nickel purity of $99.9 \%$ and $99.25 \%$ for the $10 \mathrm{~mm}$-diameter rod and $8 \mathrm{~mm}$-diameter rod, respectively. Whereas the wire is $99.9 \%$ nickel. The mechanical treatment comprised cold drawing and straight length, while the heat treatment consisted of annealing the sample in a continuous furnace. In view of the ratios of the length to the diameter, demagnetizing effect cannot be ignored. In the experiments carried out, the rods are longitudinally magnetized by the field produced by the solenoid. Magnetic poles are then induced on both ends. These, in turn, give rise to a magnetic field in a direction opposite to that of magnetization. This field, called the demagnetizing field $H_{d}$, depends on both magnetization $M$ and the geometrical dimensions [12]. The effective field $H_{\text {eff }}$ inside the sample is given by

$$
H_{e f f}=H_{s}-H_{d}=H_{s}-N_{d} \cdot M,
$$

where $H_{s}$ is the field created by the solenoid, and $H_{d}$ is expressed as the product of the demagnetizing factor $N_{d}$ and the magnetization. The factor $N_{d}$ can be calculated as a function of the ratio of the length to the diameter $r$ [13]. For the rod $10 \mathrm{~mm}$ in diameter and $180 \mathrm{~mm}$ in length, $r=18$ and $N_{d}=0.1007$, the demagnetizing field being 49.6 Oe for a saturation magnetization of $0.619 \mathrm{~T}$. 
Table I shows the results obtained following the procedure described in the previous section. The samples are referred to by their diameter in $\mathrm{mm}$. As aforementioned, $f_{1 l}$ is the frequency for the first mode and $\gamma$ the attenuation constant. Logarithmic decrement and Young's modulus are obtained by substituting $\gamma$ and $f_{1 l}$ into Eq.(8) and (9), respectively. In Table I are listed the values of $\delta$ and $E$ together with their uncertainties $u(\delta)$ and $u(E)$. The variation of Young's modulus with the magnetic field $\Delta E=E_{S}-E_{D}$, i.e. Young's modulus in the technical saturation state $E_{s}$ minus such a modulus in the demagnetized state $E_{D}$, is given in GPa along with its percentage with respect to the demagnetized state. In the same way, $\Delta \delta$ represents the absolute value of the difference between the logarithmic decrement for the saturation state $\delta_{S}$ and for the demagnetized state $\delta_{D}$; the percentage is calculated by $\Delta \delta / \delta_{D}$. Note that $\Delta \delta$ is a measure of the magnetic contribution to the damping of elastic waves.

The value of $\Delta E$ obtained for the three nickel samples is about $2 \%$. For the frequencies studied and the material tested, the difference between the values of $E$, one calculated using Eq.(9), and the other obtained without considering the term associated with the losses, is found to be negligible in comparison with the experimental errors. The value of $\delta$ in the technical saturation decreases approximately $68 \%$ with respect to that obtained with the unmagnetized samples.

Fig.4 shows the changes in both Young's modulus $E$ and logarithmic decrement $\delta$ with the magnetic fields from the demagnetized to technical saturation states for the nickel rod $10 \mathrm{~mm}$ in diameter and 180 $\mathrm{mm}$ in length.

In order to evaluate uncertainties, we have followed the ISO guide [14] for expressing measurement uncertainty. In the calculation of uncertainties, the contributions of geometrical dimensions, density, and frequency have been taken into account. For the geometrical dimensions, a digital caliper of $0.01 \mathrm{~mm}$ precision was employed. The main contribution to the frequency uncertainty $u(f)$ was the resolution obtained in the Fourier analysis (FFT), which is $10 \mathrm{~Hz}$. As far as density uncertainty $u(\rho)$ is concerned, the method described by Pratten [15] was applied to the rods $8 \mathrm{~mm}$ in diameter, $u(\rho)$ being equal to 50 $\mathrm{kg} / \mathrm{m}^{3}$. For the two other samples the data supplied by the manufacturer with an uncertainty $u(\rho)$ equal to $18 \mathrm{~kg} / \mathrm{m}^{3}$ were used. This difference in the density uncertainties results in a greater $u(E)$ for the 8 mm samples.

\section{Other experiments}

Another experiment was carried out to determine $\delta$ from the analysis of the forced vibration of the sample. The aim was to compare the results with those obtained from the free vibration. Forced longitudinal vibration was induced in order to obtain the resonance curve, i.e., the amplitude of the forced oscillations 
as a function of the exciting frequency. As aforementioned, logarithmic decrement can be determined directly from the resonance frequency and the width of such a curve at half-maximum amplitude.

The experimental set-up is similar to that showed in Fig.1, but in this experiment the sample is excited using an electromagnetic vibrator designed to induce vibrations in these kind of samples [6]. The vibrator is placed, $1 \mathrm{~mm}$ away, parallel to one of the rod's bases with its revolution axis coincident with that of the rod. When a sinusoidal potential difference is applied to the vibrator, the resulting magnetic field is of the form $B_{1}+B_{0} \sin (2 \pi \nu)$, where $B_{0}$ is smaller than $B_{1}$; the sense of the field hence remains unchanged. Consequently, the rod base is acted upon by a constant longitudinal force plus a sinusoidal one, with the same frequency as the excitation, that induces the forced vibration of the sample. The ensuing vibration is detected at the central point on the base opposite to the excited one, using the aforementioned laser interferometer. Note that neither excitation nor detection methods involve contact with the sample.

Following the method described, the resonance curve was obtained for the nickel rods referred to in the previous section. The vibrator was connected to HP-3324H signal generator with a peak-to-peak maximum amplitude of $10 \mathrm{~V}$. Then, the out-of-plane component of the displacements was detected at the central point of the base and the resulting sinusoidal signal was displayed by an oscilloscope synchronized with the generator. Inside the sample there was a uniform static magnetic field $H$, whose value was changed in each experimental step. Therefore, the resonance frequency and the width at half-maximum amplitude were determined for each value of $H$.

Eq.(5) is the differential equation governing the propagation of damped longitudinal waves along the rod. In this case the boundary conditions to be satisfied are those of a rod subjected to a force at one end and free at the other, i.e., at $z=0$ tension is of the form $\tau_{z z}=\tau_{0} e^{i \omega t}$ and at the detection point, $z=l$, there are no restrictions.

In analysing the response under the harmonic excitation, we ignored the transient motion since it exponentially decays with time, then we focused our study on the steady-state response at the detection point on the end $z=l$, which for the longitudinal vibration is given by

$$
u_{z}(t)=\frac{\tau_{0}}{E \sqrt{\left(\omega_{0}^{2}-\omega^{2}\right)-\gamma^{2} \omega^{2}}} \cos (\omega t+\theta),
$$

where $\omega_{0}$ is the undamped natural frequency and $\omega$ the exciting frequency. The amplitude of Eq.(11) as a function of the driven frequency is the resonance curve. As stated in the Introduction, the resonance frequency $f_{n}$ and the width of the resonance curve at half-maximum amplitude $\Delta f_{a}$ are related by Eq.(2). When energy dissipated is small, Eq.(3) and (2) show that $\delta$ can be expressed in terms of $f_{n}$ and $\Delta f_{a}$ as

$$
\delta=\frac{\pi \Delta f_{a}}{\sqrt{3} f_{n}} .
$$


Fig.5 shows the amplitude of vibration at the detection point against frequency for a vibrating unmagnetized nickel rod $8 \mathrm{~mm}$ in diameter, the maximum amplitude occurs at $24831 \mathrm{~Hz}$. The amplitude of vibration near resonance varies sharply, but there is no discontinuity as would be found in the ideal case without losses. There is, therefore, a loss in energy in our rod. However, damping seems to be weak because the width of the curve is narrow. The apparent width of the curve is amplified due to the scale used.

In order to determine $\Delta f_{a}$, a Lorentz function is fitted to the experimental results. Such a function for the amplitude of vibration is given by

$$
y=y_{0}+\frac{2 \cdot A}{\pi} \frac{\Delta f_{a}}{4\left(f-f_{n}\right)^{2}+\Delta f_{a}^{2}},
$$

where $f$ is the frequency, $y$ the amplitude for a given frequency divided by the maximum amplitude at resonance, $y_{0}$ is the displacement for the base line, $f_{n}$ the resonance frequency, $A$ is the whole area of the curve below the base line, and $\Delta f_{a}$ is the frequency width for half-maximum amplitude. For Fig.5, the coefficients for the best fit of Eq (13) are $A=53.0 \mathrm{~V} \cdot \mathrm{Hz}, \Delta f_{a}=37.63 \mathrm{~Hz}, f_{n}=24831 \mathrm{~Hz}$, and $y_{0}=0.1030 \mathrm{~V}$. The logarithmic decrement results to be $\delta=2.75 \cdot 10^{-3}$.

The experimental results obtained for the samples in the demagnetized and saturated states are listed in Table II. This table shows the resonance longitudinal frequency $f_{n}$, the width of the resonance curve for half amplitude $\Delta f_{a}$, the logarithmic decrement $\delta$, and Young's modulus $E$ together with the magnetic contribution to the damping, $\Delta \delta$, and the $\Delta E$. Uncertainties are also included. The results refer to the nickel rods of $10 \mathrm{~mm}$ and $8 \mathrm{~mm}$ in diameter.

\section{Conclusions}

A method for studying damping and its dependence on magnetization is presented. The method is applied to nickel rods and a nickel wire. Elastic modulus and its variation with the magnetic field is also determined in the same experiment.

The methodology followed is based on examining the free vibration of a slender magnetic rod. The out-of-plane component of the displacements is detected by means of a laser speckle interferometer. The advantages of the method applied over others are: lack of interaction with the sample, high accuracy, rapidity, and that preparation of the samples is simple. Free oscillations are excited without affecting the magnetization state. Tables included show the experimental results for the nickel rods and wire tested. As expected, damping decreases with magnetization. The increment of the logarithmic decrement for the nickel samples from the saturated to demagnetized state is of the order of $68 \%$. 
Other methodology, based on the forced vibration of the sample, is also applied in order to evaluate damping and $\Delta E$. The results obtained for $\delta$ and $E$ with the nickel samples are in good agreement with those obtained with the previous methodology; the differences can be attributed to the uncertainties and higher modes generated in the free vibration. The spectrum for the free vibration, Fig.3, shows that, apart from fundamental mode, higher modes are also excited, although their amplitudes are much smaller. Whereas in the forced excitation method the sample vibrates in the fundamental mode. Therefore, the logarithmic decrement calculated with the first method integrates the losses of the three lowest modes but with the second method $\delta$ provides the losses of the fundamental one. This could account for the small differences between the values of $\delta$ obtained with the two methods. The frequency resolution with the forced vibration technique is very high, which leads to great precision. However, this method is little operative since it takes a long time to carry out an experiment. Possible motions of the sample due to the magnetic field may cause the sample to be not aligned with the interferometer; therefore, the alignment procedure should be followed again. Another drawback is that the vibrator used is too big to excite the wire.

\section{References}

[1] R. M. Bozorth, "Ferromagnetism", D. Van Nostrad Company Inc., New york (1951), p.p. 701.

[2] Nowick A.S. and Berry B.S., "Anelastic relaxation in crystalline solids", Ed. Academic Press, New York (1992).

[3] Trémolet de Lacheisserie E., "Magnetostriction: theory and applications of magnetoelasticity", ed. C.R.C, Boca Raton-Florida (1993).

[4] P.T. Squire, "Magnetomechanical Measurement of Magnetically soft amorphous Materials", Meas. Sci. Technol., 5, 67-81 (1994).

[5] Bayón A., Gascón F., and Varadé A., Determination of the elastic constants of isotropics solids by optical heterodyne interferometry, Journal of the Acoustical Society of America, Vol. 96, No. 4, pp. 2589-2252, (1994).

[6] Bayón A., Gascón F., and Varadé A., Measurement of longitudinal vibrations in a slender rod by optical heterodyne interferometry, European Journal of Mechanics, A/Solids, Vol. 17, No. 1, pp. 167-177, (1998).

[7] J.M.Chicharro, A.Bayón, and F.Salazar, "Measurement of field-dependence elastic modulus and magnetomechanical coupling factor by optical heterodyne interferometry", Journal of Magnetism and Magnetic Materials, 202, 465-472 (1999). 
[8] Bayón A., Chicharro J.M., and Salazar F., "Simultaneous measurement of field-dependence elastic moduli by laser interferometry", Journal of Magnetism and Magnetic Materials, vol. 219, no.2, 229235 (2000).

[9] J.W. Goodman, "Statistical properties of laser speckle patterns" in Laser Speckle and Related Phenomena, Edited by J.C.Dainty (Springer-Verlag, Berlin, 1984), Chap.2, pp 9-75.

[10] A.E. Ennos, "Speckle interferometry" in Laser Speckle and Related Phenomena, Edited by J.C.Dainty (Springer-Verlag, Berlin, 1984), Chap.6, pp 203-253.

[11] Seto W.," Mechanical Vibrations", Ed. McGraw-Hill, London, (1964).

[12] B.D.Cullity, "Introduction to magnetic materials", Addison-Wesley Publishing Company, ReadingMassachusetts, 1972.

[13] S. Chikazumi and S. H. Charap, "Physics of Magnetism", Krieger Publishing Company, Florida (1994), p.p. 21-22.

[14] ISO (International Organization for Standardization), "Guide to the expresion of uncertainy in measurement", Suiza(1995)

[15] Pratten, N.A, The precise measurement of the density of small samples, J.Mater.Sci, 16, 173747(1981). 


\section{Figure captions}

Figure 1. Experimental arrangement showing all the major elements.

Figure 2. Signal of the free longitudinal vibration of a nickel rod $180 \mathrm{~mm}$ in length and $10 \mathrm{~mm}$ in diameter. The sample is in the (a) demagnetized and (b) saturated states.

Figure 3. Spectrum for the longitudinal displacement of Fig. 2a.

Figure 4. Change of elastic modulus $E$ (circles) and damping decrement $\delta$ (triangles) with the magnetic field $H$ for a nickel rod $10 \mathrm{~mm}$ in diameter and $180 \mathrm{~mm}$ in length. Results obtained following the free excitation method.

Figure 5. Amplitude curve with the forced excitation method of a nickel rod $100 \mathrm{~mm}$ in length and 8 $\mathrm{mm}$ in diameter.

\section{Tables}

Table 1. Results obtained with the free excitation method.

Table 2. Results obtained with the forced excitation method. 
Table 1: Results obtained with the free excitation method (D-Demagnetized, S-Saturated).

\begin{tabular}{|c|c|c|c|c|c|c|c|}
\hline Sample & State & $f_{1 l}(\mathrm{~Hz})$ & $\gamma$ & $\delta \pm u(\delta)$ & $E \pm u(E)(\mathrm{GPa})$ & $\Delta \delta$ & $\Delta E(\mathrm{GPa})$ \\
\hline \multirow{2}{*}{$10 \mathrm{~mm}$} & $\mathrm{D}$ & 13580 & 102 & $(3.75 \pm 0.38) \cdot 10^{-3}$ & $213.00 \pm 0.53$ & $2.48 \cdot 10^{-3}$ & 5.37 \\
& $\mathrm{~S}$ & 13750 & 35 & $(1.27 \pm 0.16) \cdot 10^{-3}$ & $218.36 \pm 0.54$ & $(67 \%)$ & $(2.5 \%)$ \\
\hline \multirow{2}{*}{$8 \mathrm{~mm}$} & $\mathrm{D}$ & 24850 & 122 & $(2.45 \pm 0.23) \cdot 10^{-3}$ & $218.0 \pm 1.5$ & \multirow{2}{*}{$1.65 \cdot 10^{-3}$} & 4.4 \\
\cline { 2 - 7 } & $\mathrm{S}$ & 25100 & 40 & $(7.97 \pm 0.92) \cdot 10^{-4}$ & $222.4 \pm 1.6$ & $(68 \%)$ & $(2.1 \%)$ \\
\hline \multirow{2}{*}{$2 \mathrm{~mm}$} & $\mathrm{D}$ & 48150 & 311 & $(3.23 \pm 0.67) \cdot 10^{-3}$ & $213.71 \pm 0.42$ & $2.18 \cdot 10^{-3}$ & 3.44 \\
\cline { 2 - 6 } & $\mathrm{S}$ & 48536 & 102 & $(1.05 \pm 0.21) \cdot 10^{-3}$ & $217.15 \pm 0.43$ & $(68 \%)$ & $(1.7 \%)$ \\
\hline
\end{tabular}

Table 2: Results obtained with the forced excitation method (D-Demagnetized, S-Saturated).

\begin{tabular}{|c|c|c|c|c|c|c|c|}
\hline Sample & State & $f_{n}(\mathrm{~Hz})$ & $\Delta f_{a}$ & $\delta \pm u(\delta)$ & $E \pm u(E)(\mathrm{GPa})$ & $\Delta \delta$ & $\Delta E(\mathrm{GPa})$ \\
\hline \multirow{2}{*}{$10 \mathrm{~mm}$} & $\mathrm{D}$ & 13638 & 24.25 & $(3.21 \pm 0.15) \cdot 10^{-3}$ & $214.82 \pm 0.50$ & $1.74 \cdot 10^{-3}$ & 5.90 \\
\cline { 2 - 8 } & $\mathrm{S}$ & 13824 & 11.17 & $(1.47 \pm 0.15) \cdot 10^{-3}$ & $220.72 \pm 0.52$ & $(55 \%)$ & $(2.7 \%)$ \\
\hline \multirow{2}{*}{$8 \mathrm{~mm}$} & $\mathrm{D}$ & 24831 & 37.63 & $(2.75 \pm 0.08) \cdot 10^{-3}$ & $217.6 \pm 1.5$ & $1.85 \cdot 10^{-3}$ & 3.10 \\
\cline { 2 - 6 } & $\mathrm{S}$ & 24957 & 12.35 & $(8.98 \pm 0.92) \cdot 10^{-4}$ & $220.7 \pm 1.5$ & $(68 \%)$ & $(1.4 \%)$ \\
\hline
\end{tabular}




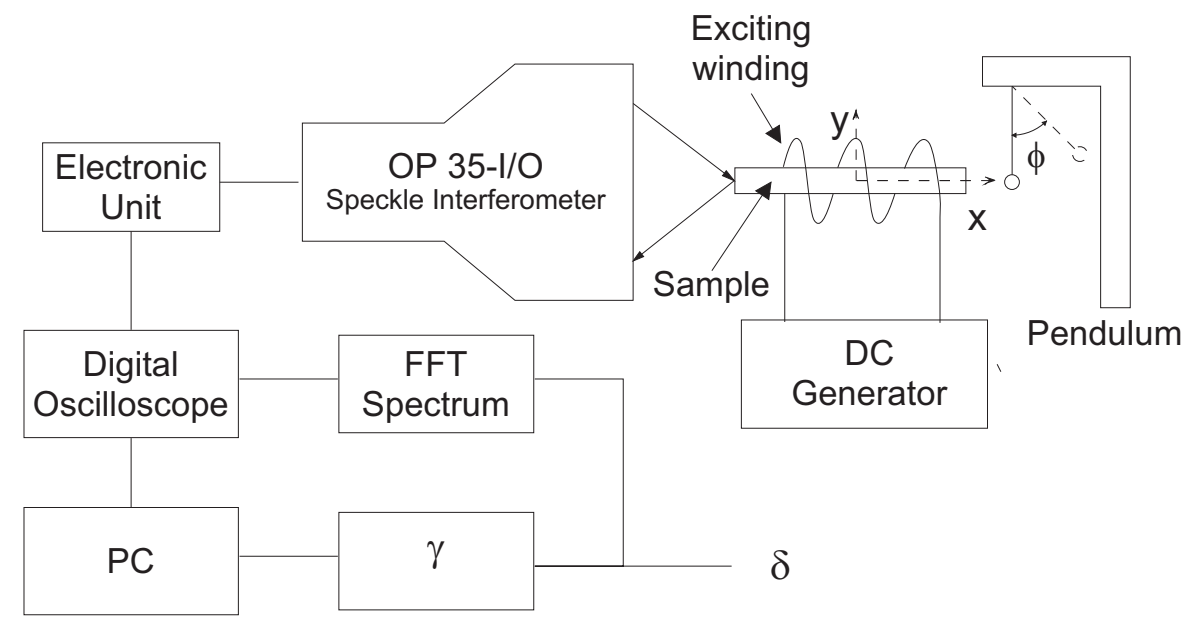

Figure 1: Experimental arrangement showing all the major elements. 


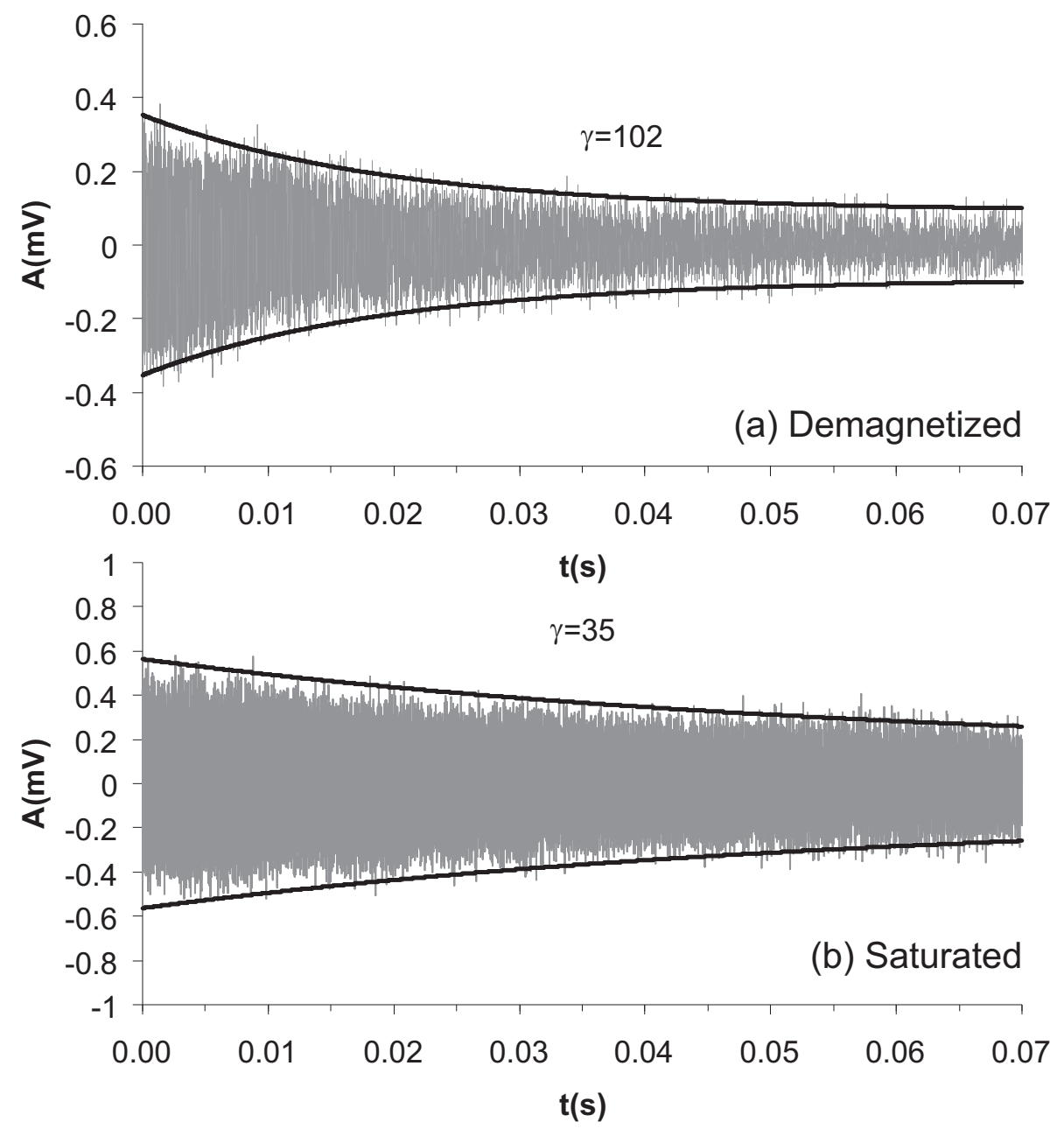

Figure 2: Signal of the free longitudinal vibration of a nickel rod $180 \mathrm{~mm}$ in length and $10 \mathrm{~mm}$ in diameter. The sample is in the (a) demagnetized and (b) saturated states. 


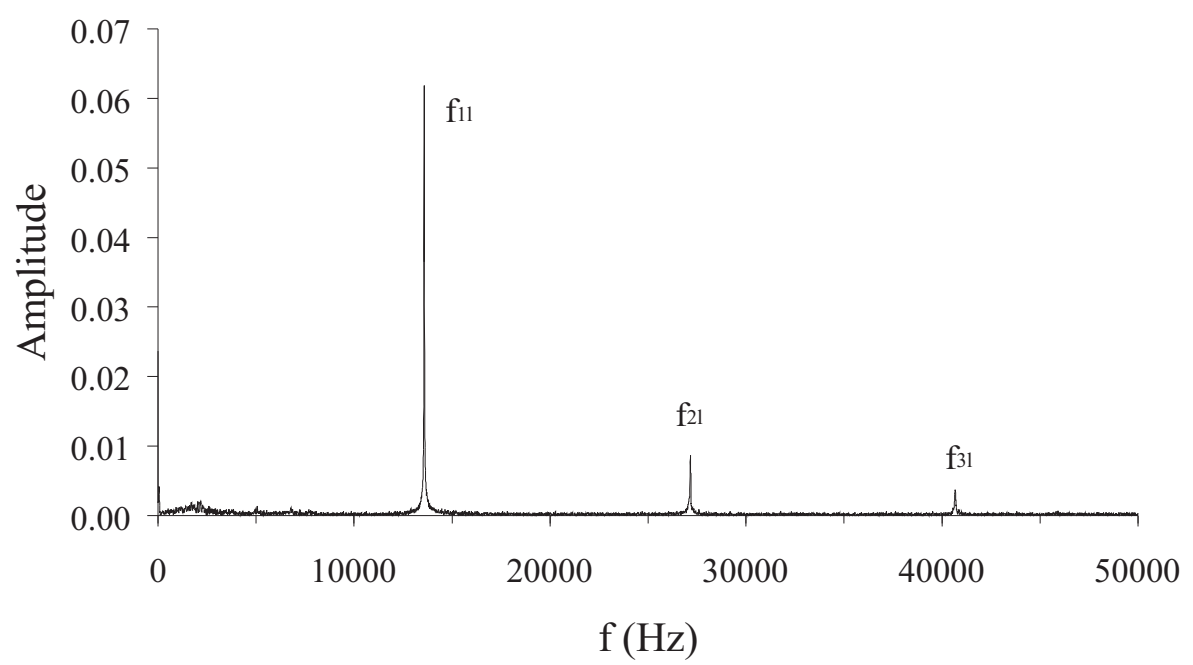

Figure 3: Spectrum for the longitudinal displacement of Fig. 2a.

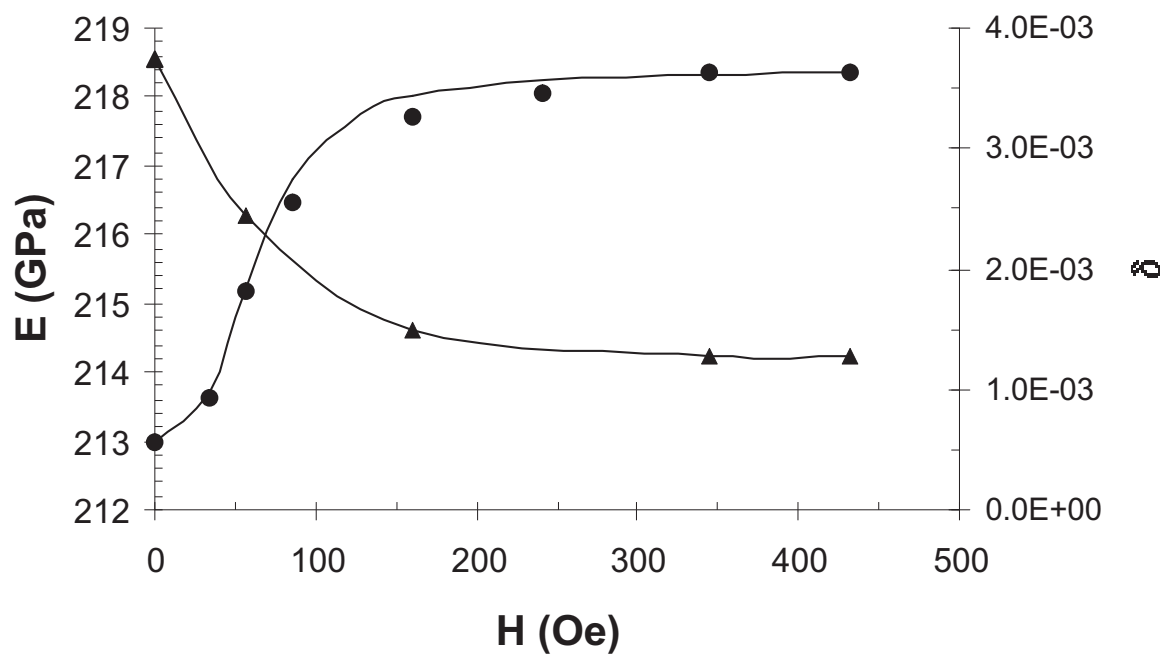

Figure 4: Change of elastic modulus $E$ (circles) and damping decrement $\delta$ (triangles) with the magnetic field $H$ for a nickel rod $10 \mathrm{~mm}$ in diameter and $180 \mathrm{~mm}$ in length. Results obtained following the free excitation method. 


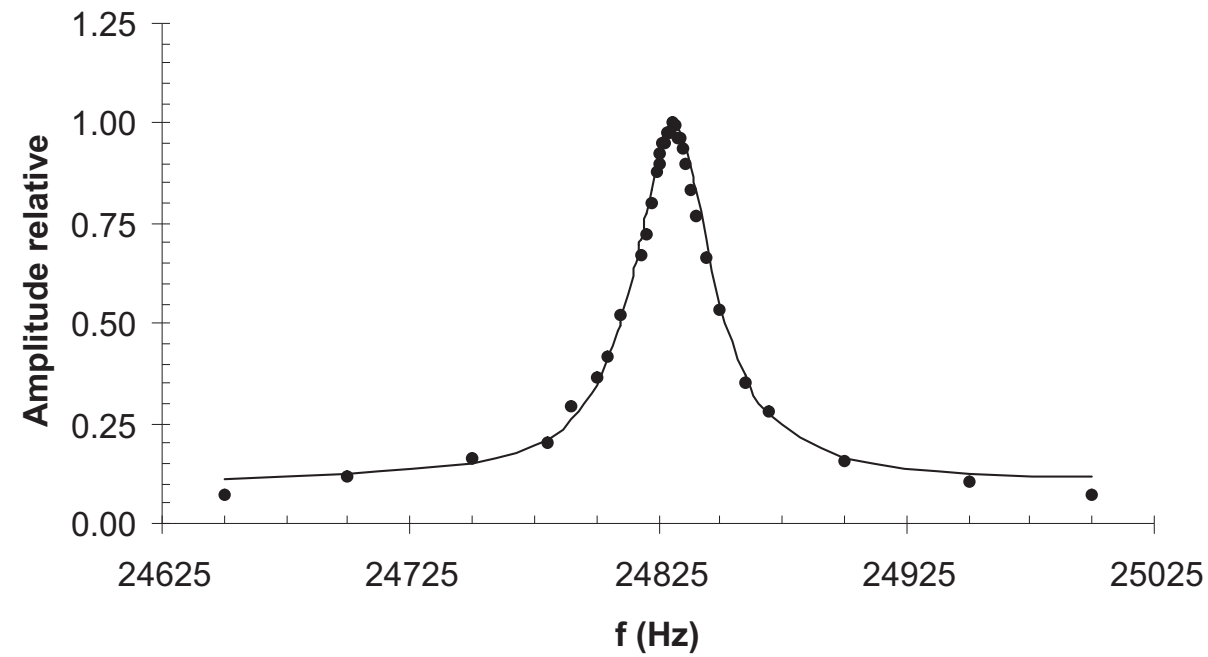

Figure 5: Amplitude curve with the forced excitation method of a nickel rod $100 \mathrm{~mm}$ in length and 8 $\mathrm{mm}$ in diameter. 